\title{
Geometric Graphs with No Self-intersecting Path of Length Three*
}

\author{
János Pach ${ }^{1}$, Rom Pinchasi ${ }^{2}$, Gábor Tardos ${ }^{3}$, and Géza Tóth ${ }^{4}$ \\ ${ }^{1}$ City College, CUNY and Courant Institute of Mathematical Sciences, \\ New York University, New York, NY 10012, USA \\ pach@cims.nyu.edu \\ 2 Department of Mathematics, \\ Massachusetts Institute of Technology, Cambridge, MA 02139, USA \\ room@math.mit.edu \\ ${ }^{3}$ Rényi Institute of the Hungarian Academy of Sciences, \\ H-1364 Budapest, P.O.B. 127, Hungary \\ tardos@renyi.hu \\ ${ }^{4}$ Rényi Institute of the Hungarian Academy of Sciences, \\ H-1364 Budapest, P.O.B. 127, Hungary \\ geza@renyi.hu
}

\begin{abstract}
Let $G$ be a geometric graph with $n$ vertices, i.e., a graph drawn in the plane with straight-line edges. It is shown that if $G$ has no self-intersecting path of length 3 , then its number of edges is $O(n \log n)$. This result is asymptotically tight. Analogous questions for curvilinear drawings and for longer paths are also considered.
\end{abstract}

\section{Introduction}

A geometric graph is a graph drawn in the plane so that its vertices are points and its edges are possibly crossing straight-line segments. We assume, for simplicity, that the points are in general position, i.e., no three points are on a line and no three edges pass through the same point. Topological graphs are defined similarly, except that now the edges are not necessarily rectilinear; every edge can be represented by an arbitrary continuous arc which does not pass through any vertex different from its endpoints. Throughout this paper, we also assume that any two edges have a finite number of common interior points and they properly cross at each of them. Clearly, every geometric graph is also a topological graph.

Using this terminology, the fact that every planar graph with $n$ vertices has at most $3 n-6$ edges can be rephrased as follows: any topological graph with $n$ vertices and more than $3 n-6$ edges must have two edges that cross each other. This result is tight even for geometric graphs.

\footnotetext{
* Work on this paper by János Pach and Rom Pinchasi has been supported by NSF grant CCR-00-98246, by PSC-CUNY Research Award 63382-0032. Work by János Pach and Gábor Tardos has also been supported by Hungarian Science Foundation grant OTKA T-032452. Work by Géza Tóth has been supported by Hungarian Science Foundation grant OTKA T-038397.
} 
In the mid-sixties Avital and Hanani AH66], Erdős, and Perles initiated, later Kupitz [K79] and many others continued the systematic study of extremal problems for geometric graphs. In particular, they proposed the following general question. Let $H$ be a so-called forbidden geometric configuration or a class of forbidden configurations. For example, $H$ may consist of $k$ pairwise crossing edges or may be the class of all configurations of $k+1$ edges, one of which crosses all the others, etc. What is the maximum number of edges that a geometric graph with $n$ vertices can have without containing any forbidden subconfiguration? If $H$ consists of $k=2$ (pairwise) crossing edges, then, according to the previous paragraph, the answer is $3 n-6$. For $k=3$, this maximum is linear in $n$ (see [AAPPS97]), but for larger values of $k$ the best known bound due to Valtr is only $O(n \log n)$ [V98. It is an exciting open problem to decide whether one can get rid of the logarithmic factor here. If $H$ is the class of all configurations consisting of $k+1$ edges, one of which crosses all the others, then the maximum number of edges is equal to $(k+2)(n-3)$, provided that $k=1,2,3$, and the maximum is $O(\sqrt{k} n)$ for large values of $k$ (cf. [PT97]). For a survey of many similar results in Geometric Graph Theory, consult [P99].

The above questions can also be regarded as geometric analogues of the fundamental problem of Extremal Graph Theory [B78]: determine the maximum number of edges of all $K$-free graphs on $n$ vertices, i.e., all graphs which do not contain a subgraph isomorphic to a fixed graph $K$. Denote this maximum by $\operatorname{ex}(n, K)$.

In the present note, we consider the special instance of the above question when $H$ consists of all self-intersecting straight-line drawings of a fixed graph $K$. In other words, what is the maximum number $\operatorname{ex}_{\mathrm{cr}}(n, K)$ of edges that a geometric graph with $n$ vertices can have, if it contains no self-intersecting copy of $K$ ? Obviously, we have $\operatorname{ex}_{\mathrm{cr}}(n, K) \geq \operatorname{ex}(n, K)$, because if a graph contains no copy of $K$, then it cannot contain a self-intersecting copy either. Therefore, if $K$ is not a bipartite graph, then $\operatorname{ex}_{\mathrm{cr}}(n, K)$ is quadratic in $n$. The question is more exciting for bipartite planar graphs. What happens if $K=P_{k}$ (or $K=C_{k}$ ), a path (or a cycle) of (an even) length $k$ ? The case where $K=C_{4}$ is discussed in PR02.

We analyze the case when $K=P_{3}$. The corresponding graph property is a relaxation of planarity: the geometric graphs satisfying the condition are allowed to have two crossing edges, but if this is the case, no endpoint of one of these edges can be joined to an endpoint of the other. Is it still true that the number of edges of such geometric graphs is $O(n)$ ? The following theorem provides a negative answer to this question.

Theorem 1. The maximum number of edges of a geometric graph with $n$ vertices, containing no self-intersecting path of length 3 , satisfies

$$
\operatorname{ex}_{\mathrm{cr}}\left(n, P_{3}\right) \leq c n \log n
$$

for a suitable constant c. Apart from the value of the constant, this bound cannot be improved. 
The proof of this result (presented in three different versions in the next three sections) applies to a slightly more general situation. Theorem 1 remains true for topological graphs whose edges are continuous functions defined on subintervals of the $x$-axis, i.e., every line perpendicular to the $x$-axis intersects each edge in at most one point. The topological graphs satisfying this condition are usually called $x$-monotone.

On the other hand, a construction in Section 3 shows that Theorem 1 cannot be improved even for geometric graphs all of whose edges are crossed by a straight line.

What happens if we drop the requirement of $x$-monotonicity? We do not have any example of a topological graph with $n$ vertices and more than constant times $n \log n$ edges, in which every path of length 3 is simple, i.e., non-self-intersecting. The best upper bound we have is the following.

Theorem 2. The maximum number of edges of a topological graph with $n$ vertices, containing no self-intersecting path of length 3 , is $O\left(n^{3 / 2}\right)$.

As was pointed out by Tutte [T70], parity plays an important role in determining the possible crossing patterns between the edges of a topological graph. This may well be a consequence of the Jordan Curve Theorem: every Jordan arc connecting an interior point and an exterior point of a simple closed Jordan curve must cross this curve an odd number of times. In particular, Tutte showed that every topological graph with $n$ vertices and more than $3 n-6$ edges has two edges that not only cross each other, but (i) they cross an odd number of times, and (ii) they do not share an endpoint. (See also H34.)

This may suggest that Theorem 2 and perhaps any other bound of this type can be sharpened as follows.

Theorem 3. The maximum number of edges of a topological graph with $n$ vertices, containing no path of length 3 whose first and last edges cross an odd number of times, is $O\left(n^{3 / 2}\right)$.

In Section 5 we prove this stronger statement. Somewhat surprisingly (to the authors), it turns out that this last result is asymptotically tight. More precisely, in Section 6 we establish

Theorem 4. Let $G$ be a bipartite graph on $n$ vertices, containing no cycle of length 4 . Then $G$ can be drawn in the plane as an $x$-monotone topological graph with the property that any two edges belonging to a path of length 3 cross an even number of times.

It is well known that there are $C_{4}$-free bipartite graphs of $n$ vertices and at least constant times $n^{3 / 2}$ edges (see e.g. B78]).

In Section 7 , we consider geometric and $x$-monotone topological graphs with no self-intersecting path of length five. In this case, Theorem 9 provides a slightly stronger bound on the number of edges than those obtained for graphs with no self-intersecting $P_{3}$. We do not believe that Theorem 9 is tight. However, a recent 
construction of Tardos [T02] shows that $\operatorname{ex}_{\mathrm{cr}}\left(n, P_{k}\right)$ is superlinear in $n$, for any fixed value $k \geq 3$.

In the final section, we discuss a few related results and open problems.

\section{A Davenport-Schinzel Bound for Double Arrays}

In this section, we discuss the special case of Theorem 1 when $G$ is a bipartite geometric (or $x$-monotone topological) graph, whose vertices are divided by the $y$-axis into two classes, $A$ and $B$, and all edges of $G$ run between these classes. We assume, for simplicity, that no two edges of $G$ cross the $y$-axis at the same point.

Let $a_{1} b_{1}, a_{2} b_{2}, \ldots, a_{m} b_{m}$ be the edges of $G$ listed from top to bottom, in the order of their intersections with the $y$-axis, where $a_{i} \in A$ and $b_{i} \in B$ for every $i$. Consider the corresponding double array $(2 \times m$ matrix $)$

$$
M=\left(\begin{array}{cccc}
a_{1} & a_{2} & \ldots & a_{m} \\
b_{1} & b_{2} & \ldots & b_{m}
\end{array}\right)
$$

It is easy to verify that if $G$ is a geometric graph (or an $x$-monotone topological graph) without any self-intersecting path of length three, then the corre-

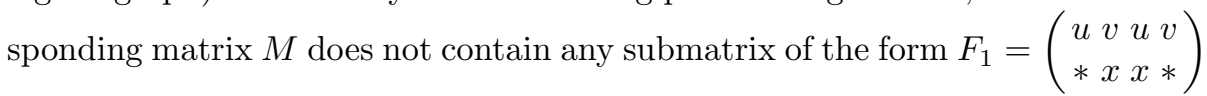

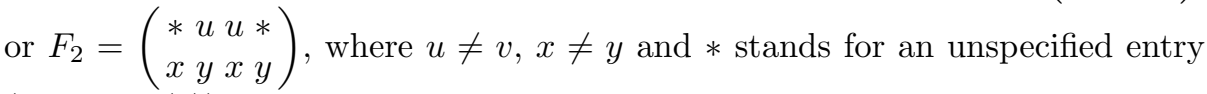
(see Fig. 1(a)).

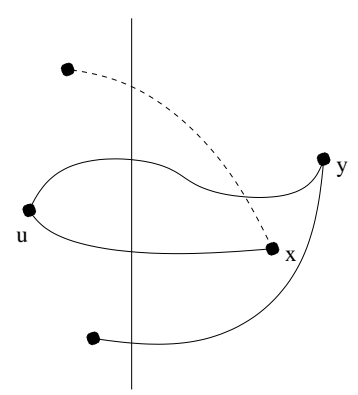

(a)

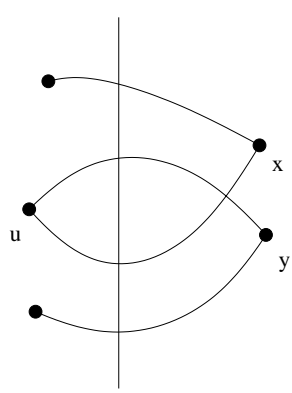

(b)

Fig. 1. (a) $F_{2}$ is forbidden, (b) not necessarily forbidden if adjacent edges may cross

In what follows, we show that if a $2 \times m$ matrix $M$ having at most $n$ distinct entries does not contain any forbidden submatrix of the above two types, then its number of columns is $O(n \log n)$. Therefore, the number of edges of $G$ is at most $O(n \log n)$, as required by Theorem 1. 
If $G$ is an $x$-monotone topological graph whose adjacent edges are allowed to cross, and we only require that the first and last edges of every path of length three must be disjoint, then the situation is slightly more complicated, because $M$ may contain submatrices of the above forms (see Fig. 1(b)). However, in this case the following $2 \times 6$ submatrices are forbidden:

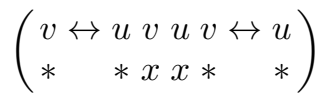

and

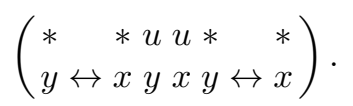

Here the signs $\leftrightarrow$ indicate that the order of the first two columns and the order of the last two columns are not specified.

Theorem 5. Let $M$ be a $2 \times m$ matrix with at most $n$ distinct entries, all of whose columns are different. If $M$ has no $2 \times 6$ submatrix of types (11) or (2), then $m \leq 17 n \log _{2} n$.

It follows from the construction at the end of Section 3, that the bound in Theorem [5 is tight apart from the value of the constant. In fact, for any $n$ there exist a $2 \times m$ matrix with at most $n$ distinct entries having neither $F_{1}$ nor $F_{2}$ as a submatrix with $m \geq n \log _{2} n / 4$.

Proof. We need some definitions. Let

$$
M=\left(\begin{array}{cccc}
a_{1} & a_{2} & \ldots & a_{m} \\
b_{1} & b_{2} & \ldots & b_{m}
\end{array}\right)
$$

For any $1 \leq i \leq m$, we say that $a_{i}$ is a leftmost (or rightmost) entry if $a_{k} \neq a_{i}$ for every $k<i$ (or $k>i$, resp.). Accordingly, $a_{i}$ is called a second leftmost (or second rightmost) entry if $a_{k}=a_{i}$ for precisely one index $k<i$ (or precisely one index $k>i$, resp.). Analogous terms are used for the entries $b_{i}$ in the second row of $M$.

A set of consecutive columns of $M$ is called a block. A block is said to be pure if all elements in the first row of the block are distinct and the same is true for the elements in the second row.

Assume the columns of $M$ are partitioned into $l$ pure blocks. Consider now two consecutive pure blocks, $B_{1}$ and $B_{2}$, consisting of the columns $i+1, i+2, \ldots, j$ and $j+1, j+2, \ldots, k$, resp., for some $0 \leq i<j<k \leq n$. Suppose that there is an element which appears in the first row of $B_{1}$ as well as in the first row of $B_{2}$. That is, $a_{p}=a_{q}$ for some $i<p \leq j$ and $j<q \leq k$. We claim that either $b_{q}$ is a leftmost, second leftmost or rightmost entry, or $b_{p}$ is a rightmost, second rightmost or leftmost entry. Indeed, otherwise, using the fact that $b_{q}$ is neither a leftmost nor a second leftmost entry, we obtain that there exists an index $r \leq i$ such that $b_{r}=b_{q}$. Since $b_{q}$ is not a rightmost entry, there is an index $s>k$ such that $b_{s}=b_{q}$. Similarly, in view of the fact that $b_{p}$ is neither a rightmost nor a second rightmost entry, we can conclude that $b_{s^{\prime}}=b_{p}$ for some $s^{\prime}>k$. Since 
$b_{p}$ is not leftmost, there is a $r^{\prime} \leq i$ such that $b_{r^{\prime}}=b_{p}$. Observe that now the columns $r, r^{\prime}<p<q<s, s^{\prime}$ form a forbidden submatrix of type

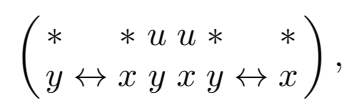

a contradiction.

A symmetric argument shows that if $b_{p}=b_{q}$ for some $i<p \leq j$ and $j<$ $q \leq k$, then either $a_{q}$ is a leftmost, second leftmost or rightmost entry, or $a_{p}$ is a rightmost, second rightmost or leftmost entry. Thus, if we delete from $M$ (and from its block decomposition) every column whose upper or lower element is a leftmost, second leftmost, rightmost, or second rightmost entry, the union of the remainders of any two consecutive blocks becomes pure.

There are at most $n$ distinct entries, each may appear in the first row and in the second row, so the number of deleted columns is at most $8 n$. The resulting matrix $M^{\prime}$ can be decomposed into $\lceil l / 2\rceil$ pure blocks. Repeating this process at most $\left\lceil\log _{2} l\right\rceil$ times, we end up with a matrix consisting of at least $m-8 n\left\lceil\log _{2} l\right\rceil$ columns that form a single pure block. Thus, we have

$$
m-8 n\left\lceil\log _{2} l\right\rceil \leq n
$$

Applying the above procedure to the initial partition of $M$ into $l=m$ pure blocks, each consisting of a single column, the upper bound follows.

For many other Davenport-Schinzel type results for matrices, consult [FH92].

As we have pointed out before, the last theorem implies that every geometric or $x$-monotone topological graph with $n$ vertices and no path of length three whose first and last edges cross each other, has at most constant times $n \log n$ edges, provided that all of its edges can be stabbed by a line. Thus, we immediately obtain

Corollary 1. The maximum number of edges of an $x$-monotone topological graph with $n$ vertices, containing no path of length 3 whose first and last edges cross, is $O\left(n \log ^{2} n\right)$.

This result is slightly weaker than the bound in Theorem 1.

\section{Proof of Theorem 1}

We prove the following more general statement.

Theorem 6. Let $G$ be an $x$-monotone topological graph of $n$ vertices, which has no self-intersecting path of length 3 . Then $G$ has at most constant times $n \log n$ edges.

We assume without loss of generality that no two edges that share an endpoint cross each other. Otherwise, the two non-common endpoints of these edges must be of degree 1 or 2 , because $G$ has no self-intersecting path of length 3 . So 
we can delete these endpoints, and complete the argument by induction on the number of vertices.

It will be convenient to use the following terminology. If a vertex $v$ is the left (resp. right) endpoint of an edge $e$, then $e$ is said to be a right (resp. left) edge at $v$. It follows from our assumption on adjacent edges that the left and the right edges at a given vertex can be ordered from bottom to top.

Let $e_{1}=v u_{1}$ and $e_{2}=v u_{2}$ be two right edges at a vertex $v$ such that the $x$-coordinate of $u_{1}$ is at most as large as the $x$-coordinate of $u_{2}$. We define the right triangle determined by them as the bounded closed region bounded by $e_{1}$, a segment of $e_{2}$, and a segment of the vertical line passing through $u_{1}$. The vertex $v$ is called the apex of this triangle. Analogously, we can introduce the notion of left triangle.

Construct a sequence of subgraphs $G_{0}, G_{1}, G_{2}, \ldots$ of $G$, as follows. Let $G_{0}=$ $G$. If $G_{i}$ has already been defined for some $i$, then let $G_{i+1}$ be the topological graph obtained from $G_{i}$ by deleting at each vertex the bottom 2 and the top 2 left and right edges (if they exist). We delete at most 8 edges per vertex.

Claim. For any $k \geq 0$, every triangle determined by two edges of $G_{k}$ contains at least $2^{k}$ pairwise different triangles of $G$.

Proof of Claim. By induction on $k$. Obviously, for $k=0$, the claim is true, because every triangle contains itself. Assume that the claim holds for $k-1(k>$ $0)$. Consider, e.g., a right triangle $T$ in $G_{k}$, determined by the edges $e_{1}=v u_{1}$ and $e_{2}=v u_{2}$, where the $x$-coordinate of $u_{1}$ is at most as large as the $x$-coordinate of $u_{2}$. Suppose without loss of generality that $e_{1}$ lies below $e_{2}$. Using the fact that $e_{1} \in E\left(G_{k}\right)$, we obtain that at $u_{1}$ there are at least two left edges $f_{1}, f_{2} \in$ $E\left(G_{k-1}\right)$ which lie above $e_{1}$. Both of these edges must be entirely contained in $T$, otherwise we could find a self-intersecting path of length 3 . Suppose that $f_{1}$ lies below $f_{2}$.

Let $T_{1}$ and $T_{2}$ denote the left triangles with apex $u_{1}$, determined by $e_{1}$ and $f_{1}$, and by $f_{1}$ and $f_{2}$, resp. Clearly, $T_{1}$ and $T_{2}$ both belong to $G_{k-1}$, and they have disjoint interiors. By the induction hypothesis, both $T_{1}$ and $T_{2}$ contain $2^{k-1}$ pairwise different triangles. It follows that $T$ contains $2^{k}$ pairwise different triangles, as required.

Now we can easily complete the proof of Theorem 6. Since every triangle is specified by a pair of edges meeting at its apex, the total number of different triangles is at most $n^{3}$. Hence, for $k>3 \log _{2} n$, the graph $G_{k}$ cannot determine any triangle, and its number of edges is smaller than $n$. On the other hand, we have that $\left|E\left(G_{k}\right)\right| \geq\left|E\left(G_{0}\right)\right|-8 k n$. Therefore, $|E(G)|=\left|E\left(G_{0}\right)\right| \leq 25 n \log _{2} n$, completing the proof of Theorem 6 .

We close this section by showing that, up to the value of the constant $c$, Theorem 1 (and hence Theorem [6, too) is best possible. Let $n=2^{k}$ be fixed. We will recursively construct a sequence of bipartite geometric graphs $G_{i}=G_{i}^{(k)}$, $i=1,2, \ldots, k$, such that $G_{i}$ has $2^{i}$ vertices, $(i+1) 2^{i-2}$ edges, and contains no self-intersecting path of length 3 . Furthermore, we will maintain the following properties for every $i$. 
1. The vertices of $G_{i}$ have distinct $x$-coordinates, which are all integers in the closed intervals $\left[-2^{k},-2^{k}+2^{i}-1\right]$ and $\left[0,2^{i}-1\right]$. Vertices with $x$-coordinates in the first (resp. second) interval are called left (resp. right).

2. Every edge of $G_{i}$ connects a left vertex to a right vertex, and hence it must cross the vertical line $\left(x=-\frac{1}{2}\right)$.

3. The horizontal edges of $G_{i}$ are of length $2^{k}$ and form a perfect matching. If two vertices of $u, v \in V\left(G_{i}\right)$, are connected by a horizontal edge, than they are said to form a pair.

4. For any vertex $v$ of $G_{i}$, the order of the edges incident to $v$ according to their slopes coincides with the order according to the lengths of their projections to the $x$-axis.

Let $G_{1}$ consist of two vertices, $\left(-2^{k}, 0\right)$ and $(0,0)$, connected by an edge. Obviously, this meets the requirements.

Assuming that we have already constructed $G_{i}$ for some $i$, we show how to obtain $G_{i+1}$. Let $G_{i}^{\prime}$ denote the translate of $G_{i}$ by a vector $\left(2^{i-1}, Y_{i}\right)$, where $Y_{i}$ is a very large positive integer to be specified later. Let $G_{i+1}$ be the union of $G_{i}$ and $G_{i}^{\prime}$, together with the following $2^{i-1}$ "new" edges: connect every left vertex $v \in V\left(G_{i}\right)$ to the right vertex $v+\left(2^{k}+2^{i-1}, Y_{i}\right) \in V\left(G_{i}^{\prime}\right)$, that is, to the right vertex forming a pair with the translate of $v$. See Fig. 2.

Choose $Y_{i}$ so large that the slope of the new edges exceeds the slope of any line induced by the points of $G_{i}$ (or by the points of $G_{i}^{\prime}$ ).

We have to check that $G_{i+1}$ has the required properties. We have $\left|V\left(G_{i+1}\right)\right|=$ $2|V(G)|=2^{i+1}$ and $\left|E\left(G_{i+1}\right)\right|=2\left|E\left(G_{i}\right)\right|+2^{i-1}=(i+2) 2^{i-1}$. Properties 1, 2,3 and 4 are all inherited from $G_{i}$. To see that property 4 is maintained, it is sufficient to recall that both the slope and length of the $x$-projection of every new edge between $G_{i}$ and $G_{i}^{\prime}$ is larger than the corresponding values for the old edges.

It remains to verify that $G_{i+1}$ does not contain a self-intersecting path of length 3. Assume to the contrary that there is such a path $P$ in $G_{i+1}$, and denote its edges by $e_{1}=u v, e_{2}=v w$, and $e_{3}=w z$. Since $G_{i}$ (and thus $G_{i}^{\prime}$ ) does not contain a self-intersecting path of length 3 , at least one of these edges must run between $G_{i}$ and $G_{i}^{\prime}$. Note that there cannot be two such edges, because all edges of $G_{i+1}$ running between $G_{i}$ and $G_{i}^{\prime}$ are parallel. It is also clear that $e_{2}$ is not such an edge.

Assume, without loss of generality, that $e_{1}$ runs between $G_{i}$ and $G_{i}^{\prime}$, and that we have $u \in V\left(G_{i}\right)$ and $v \in V\left(G_{i}^{\prime}\right)$. Thus, $e_{2}$ and $e_{3}$ belong to $G_{i}^{\prime}$. As $v$ is a right vertex, $w$ must be a left vertex, and both $e_{2}$ and $e_{3}$ are to the right of $w$. Since $e_{3}$ crosses $e_{1}$, the slope of $e_{3}$ must be smaller than that of $e_{2}$. In view of property 4 , we conclude that the $x$-coordinate of $z$ is smaller than the $x$-coordinate of $v$. This implies that the slope of the line connecting $z$ and $v$ is larger than the slope of $e_{2}$, contradicting our assumption. 


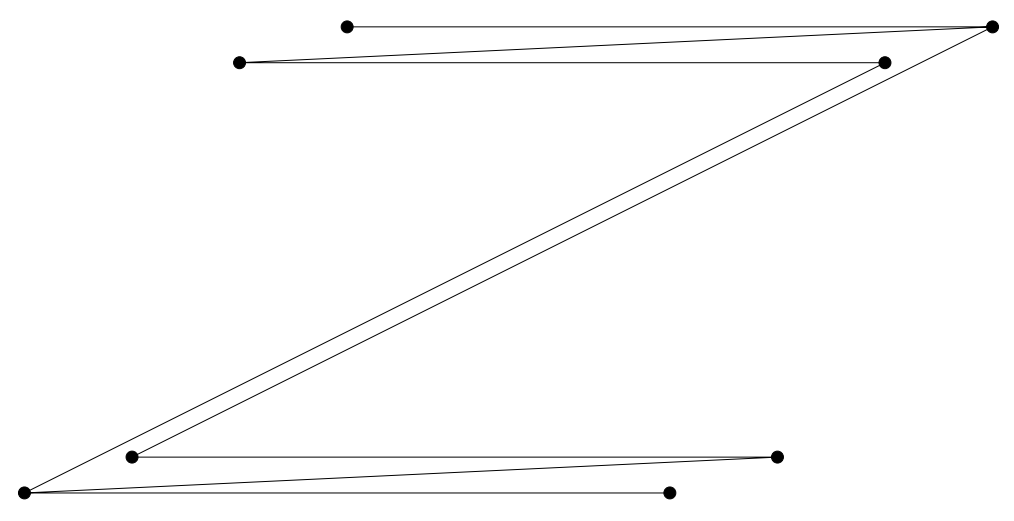

Fig. 2. The construction of $G_{i}(i=3)$

\section{A Strengthening of Theorem 6}

The aim of this section is to establish the following stronger form of Theorem 6

Theorem 7. The maximum number of edges of an $x$-monotone topological graph with $n$ vertices, containing no path of length 3 whose first and last edges cross, is $O(n \log n)$.

Proof. Let $G$ be an $x$-monotone topological graph with $n$ vertices and $m$ edges, containing no path of length 3 whose first and last edges cross. Our goal is to construct another topological graph $G^{\prime}$ with $n^{\prime}=2 n$ vertices and $m^{\prime} \geq m / 2-n$ edges, with the property that $G^{\prime}$ has no path of length 3 whose first and last edges cross, and no two adjacent edges of $G^{\prime}$ cross each other. Applying Theorem 6 to $G^{\prime}$, the statement follows.

First, we split each vertex of $G$ into into two vertices, one of them just a bit left to the other, so that every original edge $e$ becomes an edge connecting the right copy of the left endpoint of $e$ to the left copy of its right endpoint. The resulting $x$-monotone topological graph $G_{0}$ has $n^{\prime}=2 n$ vertices and $m$ edges, it has no self-intersecting path of length three, and the right endpoint of any edge of $G_{0}$ is distinct from the left endpoint of any other edge.

In the rest of this section, the length of an edge means the length of its projection to the $x$-axis, and the terms shorter and longer will be used in the same sense. We write $e=u v$ for an edge of $G_{0}$, whose left and right endpoints are $u$ and $v$, resp. We call an edge $e=u v$ long if it is the longest either among all edges $u v^{\prime}$ or among all edges $u^{\prime} v \in E\left(G_{0}\right)$. Clearly, $G_{0}$ has fewer than $n^{\prime}$ long edges. Let $e$ and $e^{\prime}$ be two edges of $G$, where $e$ is shorter than $e^{\prime}$, and we have either $e=u v$ and $e^{\prime}=u w$, or $e=v u$ and $e^{\prime}=w u$. We say that $e$ is above $e^{\prime}$ if $v$ is above $e^{\prime}$. Similarly, we say $e$ is below $e^{\prime}$ if $v$ is below $e^{\prime}$. Note that if $e$ is above or below $e^{\prime}$ then $e$ is shorter, but $e$ and $e^{\prime}$ may cross several times.

Let $e=u v$ be an edge of $G_{0}$ which is not long. By definition, there exist two edges, $e^{\prime}=u w$ and $e^{\prime \prime}=z v \in E\left(G_{0}\right)$, such that both of them are longer than 
$e$. So $e$ is either above or below $e^{\prime}$ and $e$ is also above or below $e^{\prime \prime}$. However, $e$ cannot be above both $e^{\prime}$ and $e^{\prime \prime}$. Indeed, otherwise $u$ is above $e^{\prime \prime}$ while $v$ is above $e^{\prime}$, so $e^{\prime}$ and $e^{\prime \prime}$ cross, contradicting our assumption on $G$. Similarly, $e$ cannot be below both $e^{\prime}$ and $e^{\prime \prime}$. Thus, each edge $e=u v \in E\left(G_{0}\right)$ which is not long either satisfies that $e$ is above every longer edge $u w$ and below every longer edge $z v$, or it satisfies that $e$ is below every longer edge $u w$ and above every longer edge $z v$. We can assume, by symmetry, that the former condition (which will be referred to as the monotonicity condition) holds for $m^{\prime} \geq\left(m-n^{\prime}\right) / 2=m / 2-n$ edges. Let $G_{1}$ be the subgraph of $G_{0}$ formed by these edges.

We are now in a position to define $G^{\prime}$. As an abstract graph, $G^{\prime}$ is identical to $G_{1}$. The locations of the vertices will coincide, too. For any edge $e \in E\left(G_{1}\right)$, denote by $\hat{e}$ the corresponding edge of $G^{\prime}$. We draw the edges of $G^{\prime}$ one by one, in decreasing order of length. If $e$ in $G_{1}$ is neither above nor below another edge, set $\hat{e}=e$. If $e=u v$ is above (below) at least one other edge, let $e_{-}$be the shortest edge such that $e$ is above $e_{-}$(let $e_{+}$be the shortest edge such that $e$ is below $e_{+}$, resp.). Draw $\hat{e}$ in such a way that all of its internal points lie strictly above $\hat{e}_{-}$and below $\hat{e}_{+}$(if these edges exist). Notice that, if they exist, $e_{+}$and $e_{-}$are longer than $e$, so $\hat{e}_{+}$and $\hat{e}_{-}$are already defined. We make sure during the construction that, if $e_{+}$exists, it passes above $u$, if $e_{-}$exists, it passes below $v$ (see property 2 below), and if both of them exist, they are disjoint (see property 4 below). We define $\hat{e}$ to follow $e$, except in the intervals where $\hat{e}_{+}$is below $e$ or $\hat{e}_{-}$is above $e$. In these intervals, let $\hat{e}$ run just below $\hat{e}_{+}$or just above $\hat{e}_{-}$, close enough not to intersect any further edges and going on the same side of every vertex. See Fig. 3.

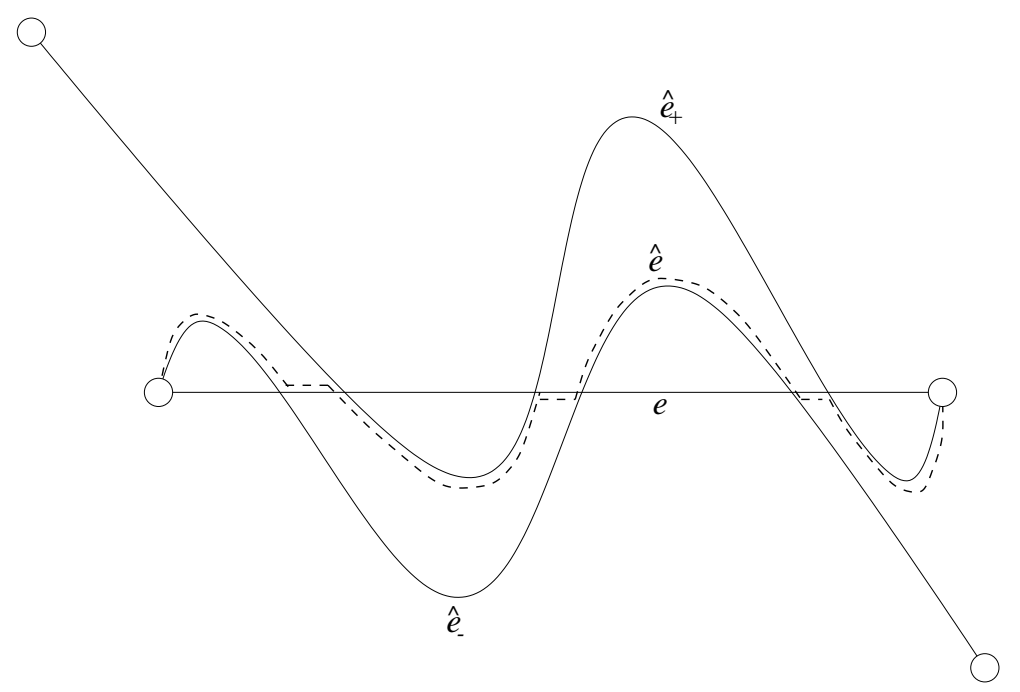

Fig. 3. The construction of the edge $\hat{e}$ in $G^{\prime}$ 
We claim that the resulting graph $G^{\prime}$ has the following properties.

1. If $e$ is below (above) $e^{\prime}$ in $G_{1}$, then every interior point of $\hat{e}$ is below (above, resp.) $\hat{e}^{\prime}$.

2. If $e^{\prime}$ is below (above) $e$ in $G_{1}$, then the endpoint of $e^{\prime}$ which is not an endpoint of $e$ is below (above, resp.) $\hat{e}$.

3. If $e, e^{\prime}$, and $e^{\prime \prime}$ form a path in $G_{1}$ and $e$ is longer than $e^{\prime}$, then $\hat{e}$ and $e^{\prime \prime}$ do not cross.

4. If $e, e^{\prime}$, and $e^{\prime \prime}$ form a path in $G_{1}$ then $\hat{e}$ and $e^{\prime \prime}$ do not cross.

We verify these properties by showing that if they hold for the partially drawn graph, they do not get violated when we add an extra edge $\hat{e}$.

(1) By the monotonicity, if there exists at least one edge $f$ such that $e$ is below $f$, then the shortest among them, $e_{+}$, must be below all others. Similarly, $e_{-}$(if exists) must be above all other edges that $e$ is above. Therefore, as property 1 has held so far, it does not get violated now, provided that $\hat{e}$ is in between $\hat{e}_{-}$ and $\hat{e}_{+}$, which is the case.

(2) Let $e=u v$ and assume that $e^{\prime}=u w$ is above $e$. By definition, $w$ is above $e$ and, by the monotonicity condition, $w$ is above $e_{-}$, if the latter exists. As property 2 has held so far, $w$ is above $\hat{e}_{-}$, so $w$ must be above $\hat{e}$. Similarly, if $e^{\prime}=z v$ is below $e$, then $z$ is below $\hat{e}$.

(3) Note that $e^{\prime}$ is above or below $e$. By symmetry, we can assume without loss of generality that $e^{\prime}$ is below $e$. By monotonicity, this means that they share their right endpoints. Here $e$ and $e^{\prime \prime}$ do not cross, as they are first and last edges of a path of length 3 , and the left endpoint of $e^{\prime \prime}$ is below $e$. So every point of $e^{\prime \prime}$ must be below $e$ or to the right of the right endpoint of $e$. If $e_{+}$exists, we can apply property 3 to the edges $e_{+}, e^{\prime}, e^{\prime \prime}$, and find that $\hat{e}_{+}$does not cross $e^{\prime \prime}$. By the construction, wherever $\hat{e}$ runs below $e$, it follows $\hat{e}_{+}$, so $\hat{e}$ is disjoint from $e^{\prime \prime}$.

(4) We consider two cases.

If both $e$ and $e^{\prime \prime}$ are shorter than $e^{\prime}$, then one of them is below and the other is above $e^{\prime}$ (monotonicity). Thus, by property $1, \hat{e}^{\prime}$ (drawn before the other two) separates $\hat{e}$ from $\hat{e}^{\prime \prime}$, so they cannot cross.

We may assume that $e$ is shorter than $e^{\prime \prime}$, so in the remaining case $e^{\prime \prime}$ is longer than $e^{\prime}$. The edge $e^{\prime}$ is below or above $e^{\prime \prime}$, and we can again assume, by symmetry, that $e^{\prime}$ is below $e^{\prime \prime}$. Applying property 3 to the path $e^{\prime \prime}, e^{\prime}, e$, we find that $e$ is disjoint from $e^{\prime \prime}$. By property 2, the left endpoint of $e$ lies below $e^{\prime \prime}$. Thus, all points of $e$ must be below $e^{\prime \prime}$ or to the right of its right endpoint. As $\hat{e}$ follows $\hat{e}_{-}$wherever it runs above $e$, it is enough to show that if $e_{-}$exists, $\hat{e}_{-}$is disjoint from $\hat{e}^{\prime \prime}$. If $e_{-}=e^{\prime}$, this follows from property 1 , otherwise, from property 4 of the initial configuration (before $\hat{e}$ has been drawn).

Observe that, by property 1 , no two adjacent edges of $G^{\prime}$ cross each other and, by property 4 , the same is true for second neighbors. Hence, we can indeed apply Theorem 6 to $G^{\prime}$, and Theorem 7 follows. 


\section{$5 \quad$ Forbidden Subgraphs - Proof of Theorem 3}

For any $k \geq 2$, let $F_{k}$ denote a graph with vertex set

$$
V\left(F_{k}\right)=\{x, y\} \cup\left\{b_{i}: 1 \leq i \leq k\right\} \cup\left\{c_{i j}: 1 \leq i<j \leq k\right\}
$$

and edge set

$$
E\left(F_{k}\right)=\left\{x b_{i}, y b_{i}: 1 \leq i \leq k\right\} \cup\left\{c_{i j} b_{i}, c_{i j} b_{j}: 1 \leq i<j \leq k\right\} .
$$

We need the following theorem, which can be obtained by a straightforward generalization of a result of Füredi [F91].

Theorem 8. For any fixed integer $k \geq 2$, let $\operatorname{ex}\left(n, F_{k}\right)$ denote the maximum number of edges of an $F_{k}$-free graph with $n$ vertices. Then we have $\operatorname{ex}\left(n, F_{k}\right)=$ $O\left(n^{3 / 2}\right)$.

Let $G$ be a topological graph with $n$ vertices, containing no path of length 3 whose first and last edges cross an odd number of times. To establish Theorem 3 . it is sufficient to verify that the abstract graph obtained from $G$ by disregarding how the edges are drawn does not have a subgraph isomorphic to $F_{4}$. In fact, it is enough to concentrate to a the subgraph $F_{4}^{\prime}$ of $F_{4}$ induceed by the vertex set $\{x, y\} \cup\left\{b_{i}: 1 \leq i \leq 4\right\} \cup\left\{c_{i j}: 1 \leq i<j \leq 3\right\}$. Notice that $F_{4}^{\prime}$ is a subdivision of $K_{5}$ : it can be obtained from $K_{5}$ by replacing four of its edges (a triangle and an edge not incident to the triangle) by paths of length two. This means that a topological graph isomorphic to $F_{4}^{\prime}$ can be also considered as a topological graph isomorphic to $K_{5}$ (simply remove the subdividing points). As $K_{5}$ is not a planar graph, any topological graph isomorphic to it must have at least one crossing. Furthermore, by Tutte's theorem [T70], there must exist two non-adjacent edges that cross an odd number of times. Thus, any topological graph isomorphic to $F_{4}^{\prime}$ has two edges that cross an odd number of times and they are either nonadjacent edges of the underlying $K_{5}$ or portions of two such edges. However, any two edges with this property can be extended to a self-intersecting path of length 3. Consequently, $F_{4}^{\prime}$ is not isomorphic to a subgraph of $G$, and Theorem 3 follows.

\section{Drawing $C_{4}$-Free Graphs - Proof of Theorem 4}

Let $G$ be a $C_{4}$-free bipartite graph with vertex set $V(G)=A \cup B$, where $A=$ $\left\{a_{1}, a_{2}, \ldots, a_{n}\right\}$ and $B=\left\{b_{1}, b_{2}, \ldots, b_{n}\right\}$. The edge set of $G$ is denoted by $E(G)$.

We now construct a drawing of $G$. Pick $2 n$ points, $a_{1}, \ldots, a_{n}, b_{1}, \ldots b_{n}$, on the $x$-axis, from left to right in this order. These points will be identified with the vertices of $G$. For every edge $a_{i} b_{j} \in E(G)$, draw an $x$-monotone arc $e_{i j}$ connecting $a_{i}$ to $b_{j}$, according to the following rules:

(i) for any $k>i$, the arc $e_{i j}$ passes above $a_{k}$ if and only if $a_{k} b_{j} \notin E(G)$;

(ii) for any $l<j$, the arc $e_{i j}$ passes above $b_{l}$ if and only if $a_{i} b_{l} \in E(G)$;

(iii) no two distinct arcs "touch" each other (internal crossings are proper). 
Notice that, unless two arcs share an endpoint, the parity of their number of intersections is determined by these rules.

Take two non-adjacent edges $a_{i} b_{j}, a_{k} b_{l} \in E(G)$ that belong to a path of length 3. We have to distinguish four different cases:

1. $i<k, j<l$, and $a_{k} b_{j} \in E(G)$;

2. $i<k, j<l$, and $a_{i} b_{l} \in E(G)$;

3. $i<k, l<j$, and $a_{i} b_{l} \in E(G)$;

4. $i<k, l<j$, and $a_{k} b_{j} \in E(G)$.

Consider the first case. By drawing rule (i), the arc $e_{i j}$ passes below $a_{k}$. By rule (ii), $e_{k l}$ passes above $b_{j}$. In view of rule (iii), this implies that $e_{i j}$ and $e_{k l}$ cross an even number of times, as required. The second case can be treated similarly and is left to the reader.

In the third case, applying rule (i), we obtain that $a_{k}$ lies above $e_{i j}$. It is sufficient to show that the same is true for $b_{l}$. At this point, we use that $G$ is $C_{4}$-free: since $a_{i} b_{j}, b_{j} a_{k}, a_{k} b_{l} \in E(G)$, we have $a_{i} b_{l} \notin E(G)$. By rule (ii), this implies that $b_{l}$ is above $e_{i j}$, as required. The last case follows in the same way, by symmetry.

So far we have checked that in our drawing any two non-adjacent edges cross an even number of times. It is not hard to extend the same property to all pairs of edges, even if they share endpoints. To this end, we slightly modify the arcs $e_{i j}$ in some very small neighborhoods of their endpoints. Clearly, this will not effect the crossing patterns of non-adjacent pairs.

Fix a vertex $a_{i}$. Redraw the arcs $e_{i j}$ incident to $a_{i}$ so that the counterclockwise order of their initial pieces in a small neighborhood of $a_{i}$ will be the same as the order of $x$-coordinates of their right endpoints. Consider now two arcs, $e_{i j}, e_{i l},(l<j)$, incident to $a_{i}$. By rule (ii), $b_{l}$ lies below $e_{i j}$. On the other hand, after performing the local change described above, the initial piece of $e_{i l}$ will also lie below $e_{i j}$. This guarantees that $e_{i j}$ and $e_{i l}$ cross an even number of times. Repeating this procedure for each vertex $a_{i}$, and its symmetric version for each $b_{j}$, we obtain a drawing which meets the requirements of Theorem 4

\section{$7 \quad$ Paths of Length Five}

If we exclude self-intersecting paths of length five (rather than three), we can establish a slightly better upper bound on the number of edges of a geometric or $x$-monotone topological graph.

Theorem 9. Let $G$ be an $x$-monotone topological graph of $n$ vertices with no self-intersecting path of length 5 . Then $G$ has at most constant times $n \log n / \log$ $\log n$ edges.

We modify the proof of Theorem [6, and use the same notation. We call an edge a left edge at its right endpoint and a right edge at its left endpoint.

Suppose $G$ has $n m$ edges with $m \geq 8$. Construct a sequence of subgraphs $G, G_{0}, G_{0}^{\prime}, G_{0}^{\prime \prime}, G_{1}, G_{1}^{\prime}, G_{1}^{\prime \prime}, G_{2}, \ldots$ of $G$, as follows. Let $G_{0}$ be the topological 
graph obtained from $G$ by deleting each vertex of degree at most $\frac{m}{2}$. Notice that no two edges of $G_{0}$ that share an endpoint cross each other. Otherwise, since all degrees are at least 5 , those two edges could be extended to a crossing path of length 5 . Thus, the left and the right edges at a vertex are naturally ordered from top to bottom. Similarly, no path of length 3 or 4 is self-intersecting.

1. If $G_{i}$ has already been defined for some $i$, let $G_{i}^{\prime}$ denote the topological graph obtained from $G_{i}$ by deleting the bottom and the top left and right edges at each vertex (if they exist). We delete at most four edges per vertex.

2. If $G_{i}^{\prime}$ has already been defined for some $i$, let $G_{i}^{\prime \prime}$ denote the topological graph obtained from $G_{i}^{\prime}$ by deleting the bottom and the top left and right edges at every vertex (if they exist). We delete at most four edges per vertex.

3. If $G_{i}^{\prime \prime}$ has already been defined for some $i$, let $G_{i+1}$ be the topological graph obtained from $G_{i}^{\prime \prime}$ by deleting recursively each vertex of degree at most $\frac{m}{2}$, so that all vertices of $G_{i+1}$ have degree higher than $\frac{m}{2}$. Clearly, as long as the average degree of $G_{i}^{\prime \prime}$ is at least $m$, it cannot decrease in this step.

Let $a_{i}$ denote the average degree in $G_{i}$. It is easy to see that $a_{0} \geq 2 m$ and $a_{i} \geq a_{i-1}-16$ as long as $a_{i-1}-16 \geq m$. So, we have $a_{\left\lfloor\frac{m}{16}\right\rfloor} \geq m$. Therefore, $G_{\left\lfloor\frac{m}{16}\right\rfloor}$ still determines at least one (actually, many) triangle(s).

Recall that a left (right) triangle at a vertex is determined by two left (right, resp.) edges of the vertex, and it is the region bounded by one of the edges, a piece of the other edge, and a vertical interval.

It is sufficient to establish the following.

Claim. For any $0 \leq k \leq \frac{m}{16}$, every triangle determined by two edges of $G_{k}$ contains at least $\left(\frac{m}{2}-2\right)^{k}$ pairwise different triangles of $G$.

Indeed, assuming that the Claim is true, a triangle determined by $G_{\left\lfloor\frac{m}{16}\right\rfloor}$ contains at least $\left(\frac{m}{2}-2\right)^{\left\lfloor\frac{m}{16}\right\rfloor}$ triangles, and this number is at most $n^{3}$. It follows that $m \leq c \log n / \log \log n$, as required by the theorem.

Proof of Claim. By induction on $k$. Obviously, for $k=0$, the assertion is true, because every triangle contains itself. Assume that the claim holds for $k-1(k>0)$. Consider a right triangle $T$ in $G_{k}$, determined by the edges $e_{1}=v u_{1}$ and $e_{2}=v u_{2}$, where the $x$-coordinate of $u_{1}$ is at most as large as the $x$-coordinate of $u_{2}$. Suppose without loss of generality that $e_{1}$ lies below $e_{2}$. Since $e_{1} \in E\left(G_{k-1}^{\prime \prime}\right)$, there is at least one left edge, $f_{1} \in E\left(G_{k-1}^{\prime}\right)$, at $u_{1}$ above $e_{1}$. This edge, $f_{1}=w_{1} u_{1}$, must entirely be contained in $T$, otherwise we could find a self-intersecting path of length 3 . Since $f_{1} \in E\left(G_{k-1}^{\prime}\right)$, there is at least one right edge, $f_{2} \in E\left(G_{k-1}\right)$, at $w_{1}$ below $f_{1}$. Similarly, this edge, $f_{2}=w_{1} w$, must be entirely contained in the triangle determined by $e_{1}$ and $f_{1}$. Therefore, $f_{2}$ must also lie in $T$. See Fig. 4. The degree of $w$ in $G_{k-1}$ is at least $\frac{m}{2}$. In view of the fact that there is no self-intersecting path of length 5 or shorter, none of these edges can cross $e_{1}, e_{2}, f_{1}$, and $f_{2}$. Therefore, all of them are entirely inside $T$. They determine at least $\frac{m}{2}-2$ triangles with pairwise disjoint interiors, each of which contains at least $\left(\frac{m}{2}-2\right)^{k-1}$ further triangles, and we are done by induction. 


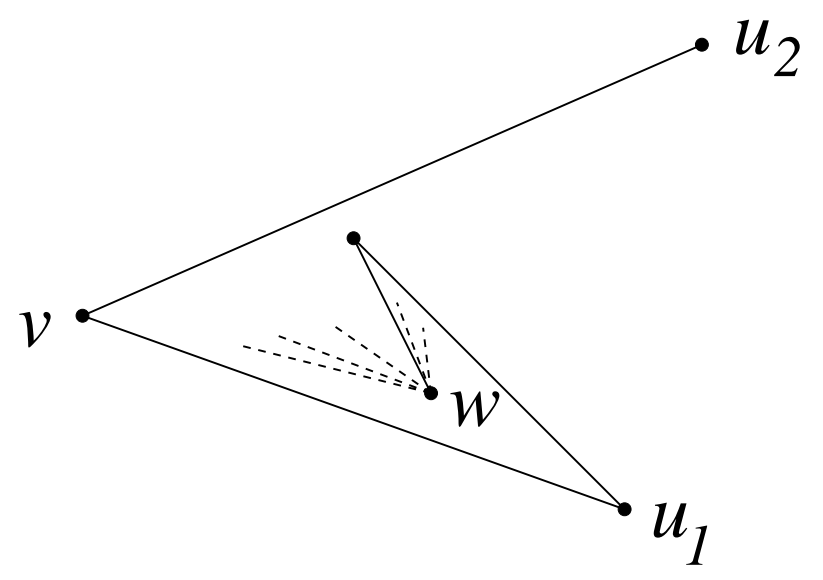

Fig. 4. The edges at $w$ are all in $T$

\section{Related Problems}

\section{A. Theorems 1 and 6 easily imply}

Corollary 2. For any tree $T$ other than a star, there exists a constant $c(T)$ such that every geometric (or $x$-monotone topological) graph $G$ with $n$ vertices and more than $c(T) n \log n$ edges contains a self-intersecting copy of T. That is, we have

$$
\operatorname{ex}_{\mathrm{cr}}(n, T) \leq c(T) n \log n .
$$

Indeed, deleting one-by-one every vertex of $G$ whose degree is smaller than $|V(T)|$, we end up with a graph $G^{\prime}$ having at most $n$ vertices and at least $(c(T) \log n-|V(T)|) n$ edges. If $c(T)$ is sufficiently large, then $G^{\prime}$ has a selfintersecting path of length 3 . Using the fact that the degree of every vertex in $G^{\prime}$ is at least $|V(T)|$, this path can be extended to a copy of $T$ in $G^{\prime}$ (and hence in $G$ ).

B. A slight modification of the proof of Theorem 1 gives

Corollary 3. For any positive integer $k$, there exists a constant $c_{k}$ with the property that every geometric graph with $n$ vertices and at least $c_{k} n \log n$ edges has two adjacent vertices, $u$ and $v$, and $2 k$ edges incident to them, $u u_{1}, u u_{2}, \ldots, u u_{k}$ and $v v_{1}, v v_{2}, \ldots, v v_{k}$, such that $u u_{i}$ crosses $v v_{j}$ for every pair $1 \leq i, j \leq k$.

C. We conjecture that Theorem 1 can be generalized to all topological graphs with with no self-intersecting path of length 3 . Recently, we have proved that if $G$ is an $x$-monotone topological graph with $n$ vertices, all of whose edges cross the $y$ axis, and $G$ has no self-intersecting path of length 4 , then $|E(G)|=O\left(n \log ^{1 / 2} n\right)$. It is interesting to note that one cannot guarantee the existence of any specific crossing pattern of a path of length 4 , even if the number of edges is at least 
$\Omega(n \log n)$. Indeed, the construction in Section 3 provides such a geometric graph with no self-intersecting path of length 3 . On the other hand, a convex, balanced, complete bipartite geometric graph, all of whose edges cross the $y$-axis, has no path of length 4 , whose only self-intersection occurs between its first and last edges.

D. Any drawing of $K_{3,3}$, a complete bipartite graph with 3 vertices in each of its classes, has two non-adjacent edges that cross each other. Clearly, any two edges belong to a cycle of length 4 , so

$$
\operatorname{ex}_{\mathrm{cr}}\left(n, C_{4}\right) \leq \operatorname{ex}\left(n, K_{3,3}\right)=O\left(n^{5 / 3}\right)
$$

This bound has been recently improved to $O\left(n^{8 / 5}\right)$ by Pinchasi and Radoičić PR02. It seems likely that the best possible bound is close to $n^{3 / 2}$.

It also follows from Theorem 8 that $\operatorname{ex}_{\mathrm{cr}}\left(n, C_{6}\right)=O\left(n^{3 / 2}\right)$, and it generalizes to topological graphs. On the other hand, we have $\operatorname{ex}_{\mathrm{cr}}\left(n, C_{6}\right) \geq \operatorname{ex}\left(n, C_{6}\right) \geq$ $c n^{4 / 3}$, for a suitable constant $c>0$ (see [BS74]). For $C_{4}$-free graphs this bound is almost tight.

Theorem 10. Let $G$ be a $C_{4}$-free geometric (or $x$-monotone topological) graph on $n$ vertices. If $G$ has no self-intersecting cycle of length 6 , then $G$ has $O\left(n^{4 / 3}\right.$ $\left.\log ^{2 / 3} n\right)$ edges.

Proof. Assume without loss of generality that the left end of an edge is not the right end of another edge in $G$. This can be achieved by splitting the vertices in two as in the proof of Theorem [7 Let $G$ have $n$ vertices and $|E(G)|=m>$ $c^{\prime} n^{4 / 3} \log ^{2 / 3} n$ edges. For $p=\frac{2 c n \log n}{|E(G)|}<1$, color randomly and independently with probability $p$ each vertex of $G$ red. Let $G^{\prime}$ be the subgraph of $G$ induced by the red vertices.

Let $i\left(G^{\prime}\right)$ denote the number of self-intersecting paths of length 3 in $G^{\prime}$. Deleting one edge from each such path, we obtain a graph with no self-intersecting path of length 3 . Thus, in view of Theorem 1, we have

$$
\left|E\left(G^{\prime}\right)\right|-i\left(G^{\prime}\right)<c\left|V\left(G^{\prime}\right)\right| \log \left|V\left(G^{\prime}\right)\right|,
$$

for some positive $c$. Taking expected values, this yields

$$
p^{2}|E(G)|-p^{4} i(G)<c p n \log n .
$$

We obtain $i(G)>\frac{|E(G)|^{3}}{8 c^{2} n^{2} \log ^{2} n}$. If $c^{\prime}$ is large enough, then $i(G)>\left(\begin{array}{l}n \\ 2\end{array}\right)$, and there must exist two self-intersecting paths of length 3 connecting the same pair of vertices. These paths cannot share an internal vertex as that would lead to a $C_{4}$. Therefore, putting them together, we get a $C_{6}$ which intersects itself at least twice. 


\section{References}

AAPPS97. P. K. Agarwal, B. Aronov, J. Pach, R. Pollack, and M. Sharir, Quasiplanar graphs have a linear number of edges, Combinatorica 17 (1997), $1-9$.

AH66. S. Avital and H. Hanani, Graphs (in Hebrew), Gilyonot Lematematika 3 (1966), 2-8.

B78. B. Bollobás, Extremal Graph Theory, Academic Press, New York, 1978.

BS74. A. Bondy and M. Simonovits, Cycles of even length in graphs, J. Combinatorial Theory, Ser. B 16 (1974), 97-105.

F91. Z. Füredi, On a Turán type problem of Erdős, Combinatorica 111 (1991), 75-79.

FH92. Z. Füredi and P. Hajnal, Davenport-Schinzel theory of matrices, Discrete Mathematics 103 (1992), 233-251.

H34. H. Hanani (C. Chojnacki), Über wesentlich unplättbare Kurven in dreidimensionalen Raume, Fundamenta Mathematicae 23 (1934), 135-142.

K79. Y. Kupitz, Extremal Problems in Combinatorial Geometry, Aarhus University Lecture Notes Series 53, Aarhus University, Denmark, 1979.

P99. J. Pach, Geometric graph theory, in: Surveys in Combinatorics, 1999 (J. D. Lamb and D. A. Preece, eds.), London Mathematical Society Lecture Notes 267, Cambridge University Press, Cambridge, 1999, 167-200.

PT97. J. Pach and G. Tóth, Graphs drawn with few crossings per edge, Combinatorica 17 (1997), 427-439.

PR02. R. Pinchasi and R. Radoičić, On the number of edges in geometric graphs with no self-intersecting cycle of length 4 , to appear.

T02. G. Tardos, On the number of edges in a geometric graph with no short self-intersecting paths, to appear.

T70. W. T. Tutte, Toward a theory of crossing numbers, J. Combinatorial Theory 8 (1970), 45-53.

V98. P. Valtr, On geometric graphs with no $k$ pairwise parallel edges, Discrete and Computational Geometry 19 (1998), 461-469. 